\title{
Congenital Malaria: First Case Report in Kuwait
}

\author{
Ali Sher ${ }^{1 *}$, Saif A. Latif ${ }^{2}$ \\ ${ }^{1}$ Infectious Diseases Hospital Laboratories, Infectious Diseases Hospital, Ministry of Health, Kuwait City, Kuwait \\ ${ }^{2}$ North Khaitan Clinic, Ministry of Health, Kuwait City, Kuwait \\ Email: *alisher02@yahoo.com, saiflatif@hotmail.com
}

How to cite this paper: Sher, A. and Latif, S.A. (2021) Congenital Malaria: First Case Report in Kuwait. Advances in Infectious Diseases, 11, 290-297. https://doi.org/10.4236/aid.2021.113026

Received: July 2, 2021

Accepted: September 7, 2021

Published: September 10, 2021

Copyright (c) 2021 by author(s) and Scientific Research Publishing Inc. This work is licensed under the Creative Commons Attribution-NonCommercial International License (CC BY-NC 4.0). http://creativecommons.org/licenses/by-nc/4.0/ (c) (7) \&) Open Access

\begin{abstract}
Background: Malaria in pregnancy poses a great health risk to the mother and her fetus and causes abortion, stillbirth, intrauterine growth retardation and low birth weight. The symptoms commonly start between 10 - 30 days of age and the symptoms mostly observed are fever, restlessness, drowsiness, jaundice, poor feeding, vomiting, diarrhea, and hepatosplenomegaly. Aim: The aim of this study was to diagnose malaria in a neonate admitted to ICU with fever, jaundice and hepatomegaly. Case Summary: A 32-day-old female child was admitted to ICU for intermittent high grade fever and rapid breathing, pallor, poor feeding, mild hepatosplenomegaly and physiological jaundice of one-week duration. The mother had malaria two years before while visiting her native country, Afghanistan and was treated with chloroquine for three days. Conclusion: High suspicion should be considered in diagnosing malaria during pregnancy to prevent congenital malaria among all neonates who present fever and splenomegaly in malaria endemic areas as well as in women from malaria endemic countries living in non-endemic areas. In this report, we describe the first case of congenital malaria in a child in non-malaria endemic Kuwait.
\end{abstract}

\section{Keywords}

Malaria, Congenital Malaria, Neonatal Malaria, Plasmodium falciparum, Plasmodium vivax

\section{Introduction}

Malaria is the third highest infectious disease killer of children in the world. Every two minutes, a child under the age of five dies from malaria. According to the 2019 world malaria report, 24 million children under five years were infected and 272,000 died worldwide in the year 2018. About 11 million pregnant women were infected with malaria infections and delivered 872,000 children with low 
birth weight during the same period [1]. The congenital malaria is defined as the detection of asexual forms of malarial parasites in a blood sample with at least one symptom of malaria within $0-7$ days, whereas in neonates between $0-28$ days provided not transmitted by a mosquito bite in non-endemic areas [2] [3] [4]. In incidental cases of malaria infection the high level of parasitaemia in asymptomatic neonates was not considered as neonatal or congenital malaria [5]. However, some newborns with congenital malaria can develop symptoms within days, weeks or months after birth [6] [7]. Congenital malaria can be acquired by the transmission of parasites from the mother to child during pregnancy or perinatally during labor [8]. In malaria endemic countries congenital malaria is considered a rare condition due to the protection from the passive transfer of maternal antibodies and fetal hemoglobin [9] [10] [11]. The incidence of congenital malaria is highly variable, between $7 \%-33 \%$, in endemic areas [12] [13]. Recently, the rate of congenital malaria has increased due to rising drug resistance, increasing virulence of the parasite, and human immunodeficiency virus (HIV) infection [14] [15]. This increase in congenital malaria is due to the availability of different highly sensitive molecular diagnostic techniques and the use of different types of samples to detect parasites. In Europe 8 cases of congenital malaria were reported in the literature [15], whereas in the USA 10 cases were reported each year since 2000 [16]. In malaria endemic areas malaria should be suspected in all neonates who present with fever and splenomegaly to avoid the antibiotics usage and prevent neonatal mortality.

\section{Case Summary}

A 32-day-old female child was admitted to ICU of the Infectious Disease Hospital, Kuwait for intermittent high grade fever and rapid breathing of one-week duration. The mother had malaria two years before while visiting her native country, Afghanistan and was treated with chloroquine $(25 \mathrm{mg}$ base/Kg body weight) for three days and came back to Kuwait, non-endemic for malaria, after three months. She has not taken radical treatment to eradicate the hypnozoites from her liver. The baby was born normally and her birth weight was $2.6 \mathrm{Kg}$. The following symptoms were observed during physical examination on admission; pallor, poor feeding, mild hepatosplenomegaly and physiological jaundice. Complete blood count showed severe anemia with hemoglobin $(\mathrm{Hb}) 7.7 \mathrm{~g} / \mathrm{L}$, hematocrit $0.253 \mathrm{~L} / \mathrm{L}, \mathrm{MCV} 78.8 \mathrm{fl}, \mathrm{MCH} 26.4 \mathrm{pg}, \mathrm{MCHC} 339 \mathrm{~g} / \mathrm{L}$, red blood cell count $3.25 \times 10^{12} / \mathrm{L}$, white blood cell count $10.5 \times 10^{9} / \mathrm{L}$ and platelet count $40 \times$ $10 \%$ L. Differential count revealed $45.8 \%$ lymphocytes, $18.5 \%$ monocytes, $33.4 \%$ neutrophils and $0.5 \%$ eosinophils (Table 1 ). The other laboratory findings include total bilirubin $30.80 \mu \mathrm{mol} / \mathrm{L}$, aspartate aminotransferase $207 \mathrm{IU} / \mathrm{L}$, alanine aminotransferase 64.0 IU/L and normal alkaline phosphatase (219.0 IU/L). C-reactive protein (CRP) was mildly elevated $(34 \mathrm{mg} / \mathrm{L})$, and serum electrolytes were within the normal range (Table 1). The mother was asymptomatic at the time of her baby's admission. 
Table 1. Hematological parameters of the infant on admission.

\begin{tabular}{ccc}
\hline & Laboratory results & \\
\hline Parameters & Observed value & Normal value \\
\hline RBC & $3.25 \times 10^{12} / \mathrm{L}(\downarrow)$ & $4.5-5.5 \times 10^{12} / \mathrm{L}$ \\
WBC & $10.5 \times 10^{9} / \mathrm{L}(\uparrow)$ & $4.0-10.0 \times 10^{9} / \mathrm{L}$ \\
Haemoglobin & $8.6 \mathrm{~g} / \mathrm{dl}(\downarrow)$ & $120-150 \times \mathrm{g} / \mathrm{dl}$ \\
Platelets & $40 \times 10^{9} / \mathrm{L}(\downarrow)$ & $150-410 \times 10^{9} / \mathrm{L}$ \\
C-reactive protein & $34 \mathrm{mg} / \mathrm{L}(\uparrow)$ & $0.0-8.0 \times \mathrm{mg} / \mathrm{L}$ \\
T. Bilirubin & $58.14 \mu \mathrm{mol} / \mathrm{L}(\uparrow)$ & $0.0-34.0 \mu \mathrm{mol} / \mathrm{L}$ \\
Conjugated Bilirubin & $34.0 \mu \mathrm{mol} / \mathrm{L}(\uparrow)$ & $<5.1 \mu \mathrm{mol} / \mathrm{L}$ \\
Unconjugated bilirubin & $24.0 \mu \mathrm{mol} / \mathrm{L}(\uparrow)$ & $1.7-17.1 \mu \mathrm{mol} / \mathrm{L}$ \\
\hline
\end{tabular}

In malaria diagnosis the microscopic examination of Giemsa stained thick and thin blood smears, and immunochromatographic (ICT) malaria test for the detection of histidine rich protein-2 (HRP2) antigen was used. The ICT assay was performed as described by following the manufacturer's instructions. The Giemsa stained peripheral blood smears of both mother and baby revealed the mixed infection of Plasmodium falciparum (rings and gametocytes) (Figure 1) and Plasmodium vivax (trophozoites and gametocytes) (Figure 2). The observed parasitemia was $0.2 \%$ in the baby's blood, whereas, very low level of parasitemia was found in the mother's blood $(<0.001)$. Rapid immunochromatographic test for histidine rich protein-2 (HRP2) (AccuBio Tech Co., Ltd. Bejing, China) was also positive for the child only (Figure 3 ). The parasite count was very low in the mother's blood sample, therefore, the ICT was found negative. The immunochromatographic test detects antigen in blood samples only if the parasite count is about 100 or more / $\mu \mathrm{l}$ of blood [17]. Antimalarial therapy with intravenous artesunate $(3 \mathrm{mg} / \mathrm{Kg}$ body weight IV at 0,12 and $24 \mathrm{hr}$ ) was initiated and followed with Coartem (one tablet as the initial dose, 1 tablet again after 8 hours, and then 1 tablet twice daily (morning and evening) for the following 2 days (total 6 tablets). The peripheral parasitemia was cleared completely and the hematological parameters became normal after treatment. The baby was followed weekly for three weeks using Giemsa stained thick and thin blood films for any residual parasites. The mother was treated with Coartem (four tablets as a single dose), 4 tablets again after 8 hours, and then 4 tablets twice daily (morning and evening) for the following 2 days (total 24 tablets). The mother was referred to the preventive department for radical treatment (chloroquine $25 \mathrm{mg} / \mathrm{Kg}$ body weight and primaquine $5 \mathrm{mg} /$ body weight weekly for 8 weeks). The infection in neonates takes place through the transmission of infected erythrocytes from the mother rather than the merozoites from the liver, therefore, the radical treatment was not required to the baby. 


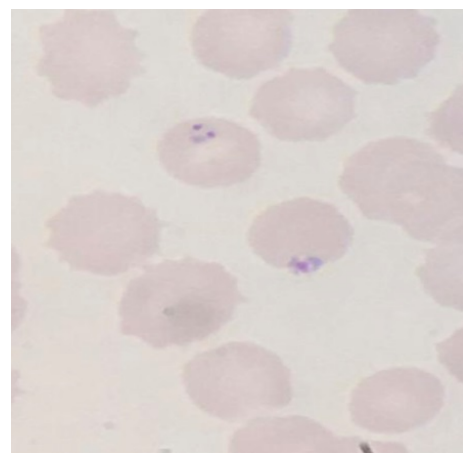

(a)

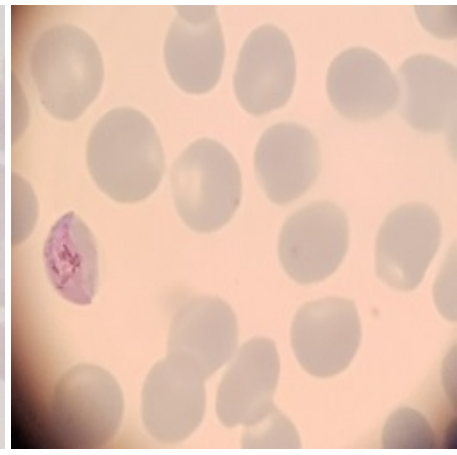

(b)

Figure 1. Child and mother's blood smears. (a) (b) Child and Mother's Giemsa stained thin blood smear showing $P$. falciparum (ring and gametocytes) within erythrocytes $(1000 \times)$.

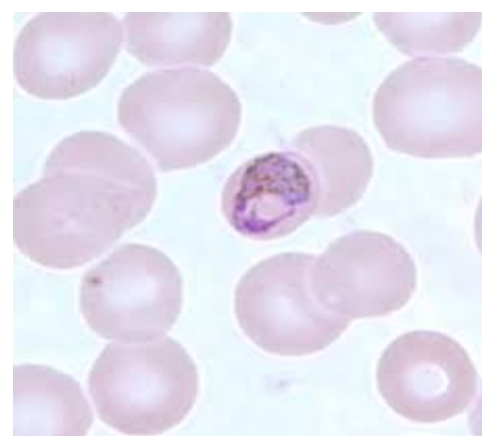

(a)

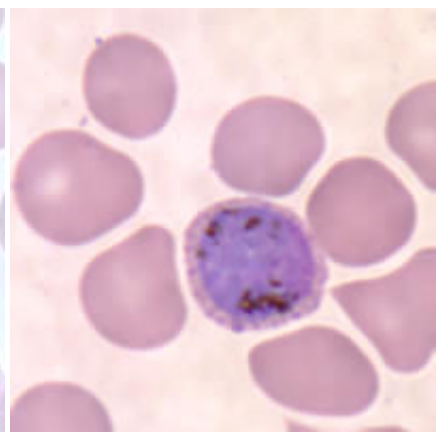

(b)

Figure 2. Child and mother's blood smears. (a) (b) Child and mother's Giemsa stained thin blood smear showing $P$. vivax (trophozoites and gametocytes) within erythrocytes (1000x).

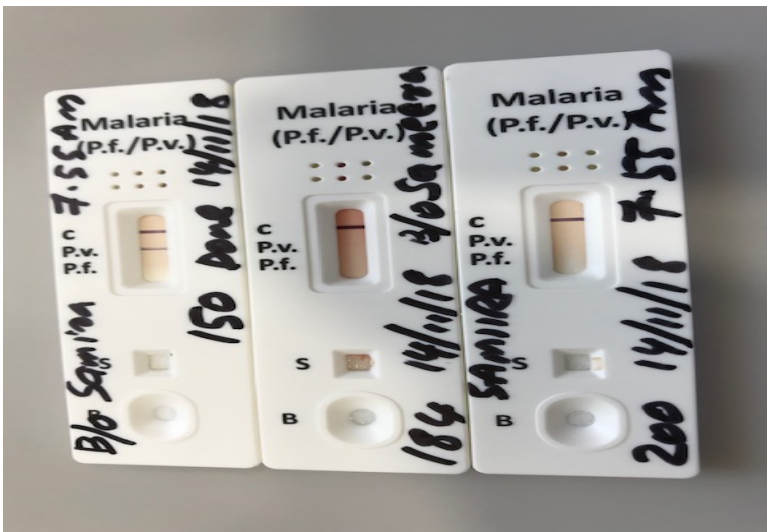

(a) (b)

(c)

Figure 3. Pattern of rapid ICT malaria test for child and her mother: ((a) before treatment; (b) after treatment for baby; (c) before treatment for mother).

\section{Discussion}

The presence of asexual stages of the parasite in cord blood at the time of deli- 
very or in the peripheral blood smear of a newly born baby in the first seven days of life is the definition of congenital malaria with at least one symptom [1]. In the Southeast Asian region Plasmodium falciparum and $P$. vivax have been reported more often as a cause of congenital malaria [2]. In Europe $P$. vivax is the leading cause of congenital malaria whereas in Indian and African subcontinent $P$. falciparum remains the leading species of malaria. The infection rate of neonatal malaria is very low, $0.3 \%$ in immune mothers and $7.4 \%$ in non-immune mothers [5] [6] [7]. Generally, one-third of women acquire the infection through placental infection during pregnancy. The clearance of infection in neonates in endemic areas is $93 \%$ due to the protective role of maternal antibodies and fetal hemoglobin in slowing the rate of parasite development. The malaria infection was rarely detected before in neonates due to lack of sensitive molecular techniques and most cases are accidentally detected on peripheral blood examination.

The non-specific clinical symptoms like, fever, anemia, and splenomegaly are common in congenital malaria [4] [5], therefore, all neonates born to mothers in malarial endemic countries or with a history of malaria during pregnancy are considered as congenital malaria and differential diagnosis of neonatal sepsis is performed.

The remarkable capability of a fetus to resist malarial infection has been demonstrated very well. The presence of the placental barrier, transfer of protective maternal antibodies, and high levels of fetal hemoglobin are the protective factors [9] [10]. The mode of congenital transmission of malaria is mostly through maternal transfusion into fetal circulation at the time of delivery or during pregnancy, direct penetration through chorionic villi, or penetration via premature rupture of the placenta [18]. Congenital malaria may transpire despite the absence of any evidence of active malarial infection in the mother during pregnancy. The history of this Afghani mother of the patient had an episode of fever during the ninth month of pregnancy which was mild, resolved spontaneously, and remained undiagnosed. The lack of maternal parasitemia and histidine rich protein-2 (HRP2) antigenemia suggests that the infection was localized to the placenta and had cleared. The disagreement between maternal peripheral blood examination/antigen testing and placental parasitization is well described in the literature [9]. The time of onset of symptoms in congenital malaria can vary from immediately after birth to few weeks, and the median age of expression has been described as 21 days [19] [20].

The prevalence of clinical congenital malaria was reported in $40.4 \%$ (95\% CI 19.6 - 67.7; 17 studies) cases till 2020. There were no significant differences between the prevalence of clinical congenital malaria in Africa $39.5 \%$ and outside Africa 56.3\% (95\% CI 17.2 - 59.5; 15 studies). There was no difference in the prevalence of clinical neonatal malaria in Africa (12.1\%) and outside Africa (12.5\%) [5] [9]. Congenital malaria presenting as neonatal sepsis and respiratory distress has been described in few reports from across the world [5]-[11].

In congenital malaria only the curative therapy is required to eradicate the plasmodium species, as the infection takes place through the transmission of in- 
fected erythrocytes from the mother rather than the merozoites from the liver, therefore, the neonate does not require the radical treatment for exo-erythrocytic stages of the parasite.

The present case was accidentally picked up on Giemsa stained peripheral blood film examination. This shows the importance of a good peripheral blood film as a part of all suspected cases of neonatal sepsis. To conclude, malaria should be suspected in all neonates born from a mother of malaria endemic areas who presented with fever and splenomegaly. Congenital malaria is not rare as it was thought to be in malaria endemic areas. Early diagnosis could prevent unnecessary antibiotics usage and could prevent neonatal mortality.

\section{Conclusion}

We report the first case of congenital malaria in a 32-day-old baby girl from an Afghani mother who presented with high grade fever and rapid breathing of one-week duration. The diagnosis of congenital malaria was confirmed by microscopy and detected the mixed infection of $P$. falciparum and $P$. vivax in Giemsa stained peripheral blood smears. The onset of symptoms and parasitemia in the patient also confirmed the diagnosis of congenital malaria. High suspicion should be considered in diagnosing malaria during pregnancy in malaria endemic areas to prevent congenital malaria.

\section{Acknowledgements}

We would like to thank all the technical staff of the hematology section of the IDH laboratories for their technical assistance.

\section{Consent for Publication}

Consent for publication was given by the ministry of health, Kuwait.

\section{Statement of Ethics}

The study was approved by the Ethics Committee for the protection of Human Subjects in Research, Ministry of Health, Kuwait.

\section{Funding}

The financial support for this study was provided by the Ministry of Health, Kuwait.

\section{Conflicts of Interest}

The authors declare no conflicts of interest regarding the publication of this paper.

\section{References}

[1] World Health Organization (2019) World Malaria Report. World Health Organization. https://apps.who.int/iris/handle/10665/330011 
[2] Menendez, C. and Mayor, A. (2007) Congenital Malaria: The Least Known Consequence of Malaria in Pregnancy. Seminars in Fetal \& Neonatal Medicine, 12, 207-213. https://doi.org/10.1016/j.siny.2007.01.018

[3] Stassijns, J., van den Boogaard, W., Pannus, P., Nkunzimana, A. and Rosanas-Urgell, A. (2016) Prevalence and Diagnostics of Congenital Malaria in Rural Burundi, a Cross-Sectional Study. Malaria Journal, 15, Article No. 443.

https://malariajournal.biomedcentral.com/articles/10.1186/s12936-016-1478-0 https://doi.org/10.1186/s12936-016-1478-0

[4] Ibhanesebhor, S.E. (1995) Clinical characteristics of Neonatal Malaria. Journal of Tropical Pediatrics, 41, 330-333. https://doi.org/10.1093/tropej/41.6.330

[5] Danwang, C., Bigna, J.J., Nzalie, R.N.T., et al. (2020) Epidemiology of Clinical Congenital and Neonatal Malaria in Endemic Settings: A Systematic Review and Meta-Analysis. Malaria Journal, 19, Article No. 312. https://doi.org/10.1186/s12936-020-03373-8

[6] Poespoprodjo, J.R., et al. (2010) Case Report: Severe Congenital Malaria Acquired in Utero. The American Journal of Tropical Medicine and Hygiene, 82, 563-565. https://doi.org/10.4269/ajtmh.2010.09-0744

[7] Lehner, P.J. and Andrews, J.A. (1988) Congenital Malaria in Papua New Guinea. Transactions of the Royal Society of Tropical Medicine and Hygiene, 82, 822-826. https://doi.org/10.1016/0035-9203(88)90006-5

[8] D’Alessandro, U., Ubben, D., Hamed, K., Ceesay, S.J., Okebe, J., Taal, M., et al. (2012) Malaria in Infants Aged Less Than Six Months-Is It an Area of Unmet Medical Need? Malaria Journal, 11, Article No. 400. https://doi.org/10.1186/1475-2875-11-400

[9] Cohen, S., McGregor, I.A. and Carrington, S. (1961) Gamma-Globulin and Acquired Immunity to Human Malaria. Nature, 192, 733-737. https://doi.org/10.1038/192733a0

[10] Billig, E.M., McQueen, P.G. and McKenzie, F.E. (2012) Foetal Haemoglobin and the Dynamics of Paediatric Malaria. Malaria Journal, 11, Article No. 396. https://doi.org/10.1186/1475-2875-11-396

[11] Shear, H.L., Grinberg, L., Gilman, J., Fabry, M.E., Stamatoyannopoulos, G., Goldberg, D.E., et al. (1998) Transgenic Mice Expressing Human Fetal Globin Are Protected from Malaria by a Novel Mechanism. Blood, 92, 2520-25526. https://doi.org/10.1182/blood.V92.7.2520

[12] Desai, M., ter Kuile, F.O., Nosten, F., McGready, R., Asamoa, K., Brabin, B., et al. (2007) Epidemiology and Burden of Malaria in Pregnancy. The Lancet Infectious Diseases, 7, 93-104. https://doi.org/10.1016/S1473-3099(07)70021-X

[13] Eki-Udoko, F.E., Sadoh, A.E., Ibadin, M.O. and Omoigberale, A.I. (2017) Prevalence of Congenital Malaria in Newborns of Mothers Co-Infected with HIV and Malaria in Benin City. Infectious Diseases, 49, 609-616.

https://doi.org/10.1080/23744235.2017.1312667

[14] Carlier, Y., Truyens, C., Deloron, P. and Peyron, F. (2012) Congenital Parasitic Infections: A Review. Acta Tropica, 121, 55-70. https://doi.org/10.1016/j.actatropica.2011.10.018

[15] Rutgers, M.M., Clemens, S.C.M., de Winter, J.P., Sloot, S.C., Jager, M.M., et al. (2017) Congenital Plasmodium vivax Malaria in a Non-Endemic Country: A Unique Case in the Netherlands. Pediatric Infectious Diseases, 2, 33.

[16] D’Avanzo, N.J., et al. (2002) Congenital Malaria as a Result of Plasmodium Malariae-North Carolina, 2000. Morbidity and Mortality Weekly Report, 51, 164-165. 
[17] Ali, S., Iqbal, J., Hira, P.R., Owaish, R.A. and Anezi, A.A. (1996) Rapid Diagnosis of Malaria by Antigen-Capture Method. Proceedings of the 10 th Scientific Congress of the Kuwait Medical Association, Kuwait City, November 1996, 46-49.

[18] Lalita, S. and Geeta, S. (2017) Placental Malaria: A New Insight into the Pathophysiology. Frontiers in Medicine, 4, Article No. 117.

[19] Preeti, R., Kaushik, M., Sunita, S., Richa, C. and Jagdish, C. (2015) Congenital Malaria in a Neonate: Case Report with a Comprehensive Review on Differential Diagnosis, Treatment and Prevention in Indian Perspective. Journal of Parasitic Diseases, 39, 345-348. https://doi.org/10.1007/s12639-013-0342-1

[20] Ravi, B., Dinesh, R. and Priti, A. (2016) Congenital Malaria Due to Plasmodium vivax Infection in a Neonate. Case Reports in Pediatrics, 2016, Article ID: 1929046.

https://doi.org/10.1155/2016/1929046 